\title{
The Design and Application of GIS Mathematical Model Database System with Meta-algorithm
}

\author{
Li-Zhijiang \\ Yishui College, Linyi University, Linyi, Shandong,276400
}

Keywords: Meta-algorithm; GIS Mathematical model database system; Design; Application

\begin{abstract}
Meta-algorithm in GIS system changes the single and fixed mode of mathematical model creation in GIS system, which adapts to the new development demand. This paper focuses on analyzing mathematical model library system and GIS mathematical model library system under meta-algorithm, classifies models, designs model logical organization structure and system model management, and meets the new demands of GIS on mathematical models. Provide positive and reliable algorithm support for GIS to make it better applied to the practice of social production.

GIS has close relationship with mathematical model. GIS functions such as data access, preliminary processing and query data, etc., all revolve data, which directly or indirectly use more mathematical models. Fixed static mathematical model in the past is a fixed static form. Model management is relatively single, giving priority to application. The mathematical model now can't meet the new development of GIS, and GIS mathematical model database system design and application under meta-algorithm can well meet the new development.
\end{abstract}

\section{Meta-ALgorithm}

There are lots of GIS algorithms, which are complex and changeable, but overall they can be generalized down into several basic algorithms combination. These are "meta-algorithms". "Metaalgorithm" is now widely used in scientific research and in various fields of social productions, and GIS is no exception. When using this algorithm in GIS field, there should be no principle of "the thinner, the better" selection algorithm, and should reasonably select algorithm considering the actual demand. Spatial analysis in GIS system, for example, involves a large number of spatial data probability algorithms. There is a need to establish corresponding algorithm, referring to as "extended algorithm". Actually meta-algorithm has some specific classification. The first is basic meta-algorithm. Elementary function calculations often use this kind of algorithm, such as trigonometric functions, logarithmic function, exponential function and simple addition, subtraction, multiplication, and division, etc. in basic elementary function. Some elementary functions often use this algorithm. Extension meta-algorithm is the second. GIS often uses this with greater use frequency. But it is a kind of basic algorithm beyond the basic meta-algorithm. Extended metaalgorithm is often used in the process of GIS data acquisition, processing and analysis. Spatial analysis in theory of probability and fuzzy mathematics content will also use this extension metaalgorithm to reduce repeated computation effectively, which will also facilitate the building of the new algorithm model [1].

\section{MATHEMATICAL MODEL LIBRARY SYSTEM AND GIS MATHEMATICAL MODEL DATABASE SYSTEM UNDER META-ALGORITHM}

\section{A. Mathematical model library system under meta-algorithm}

In accordance with relevant model theory, relevant personnel put forward the concept of "metaalgorithm". Because the oriented objects of GIS system has abstractness. From the mathematical sense of mathematical model, it can be classified into basic and universal algorithm models. Then model atomic model size and expansion express can determine the possibility. Meta-algorithm can be used as the control scale of the atomic model size. Because this kind of algorithm has characteristics like generality, basically undividable and scientific, it has very good effect in terms of control model. When GIS selects algorithm model, analyze a large number of mathematical 
model structures in GIS90 in detail, summarize meta-algorithm, and the following classification algorithms can be got: the first is basic element algorithm mathematical model, which is mainly used for elementary function calculation. The second is regular mathematical model, which are mainly regular mathematical models serving basic meta-algorithm mathematical model [2]. For example, when calculate arrays, there are problems like array length, differential, and maximum. Using basic meta-algorithms can't realize the calculation in these aspects, and we need to convert it to meta-algorithm mathematical model for calculation and processing.

\section{B. GIS mathematical model database system under meta-algorithm}

GIS mathematical model library system's foundation under meta-algorithm is meta-algorithm model. On this basis, establish a mathematical rapid development platform to better manage, maintain and apply GIS mathematical model resources to provide relevant staff and user management tool. Under meta-algorithm, this kind of mathematical model system, compared with traditional model base structure, is roughly the same. It is made up of four parts: model library, management system, administrators, and application. GIS mathematical model library system functions under meta-algorithm are mainly the following kinds: 1 . unified management of a large number of mathematical models in GIS to achieve the unified and effective management of GIS mathematical model. Management functions mainly include management control model library, and management contents mainly are expression, reading, storage, classification, and generate new models, and other functions. 2. Provide users with rapid development of corresponding mathematical model database tools. This algorithm application in mathematical model can meet the different needs of users in creating mathematical model library, and mathematical model building method under meta-algorithm is very different from the traditional mathematical model building. It can really reasonably use and control mathematical model and well manage the existing model. It provides support for the construction of a new mathematical model, so that GIS has a reliable and efficient algorithm to support. 3. External system calls have uniform interface. In the beginning, system models have corresponding interfaces, and external systems can also call the interfaces according to actual condition, and it can meet the needs of specific mathematical model function for practical application.

\section{GIS MATHEMATICAL MODEL LIBRARY SYSTEM DESIGN UNDER META-ALGORITHM}

\section{A. Model classification}

Meta-algorithm model can easily achieve effective management by first classifying algorithm models. Establish the corresponding architecture. According to meta-algorithm calculation and use characteristics, the classifications can be: 1 . Consider meta-algorithm model parameters and operation, which can be divided into monocular and binocular meta-algorithm. The monocular meta-algorithm includes cosine, absolute value, etc.; Binocular meta-algorithm includes add, subtract, multiply and divide and index operation, etc. 2. Algorithm model based on the combination character types and operation results can be divided into four types. Meta-algorithm results in addition are likely to be a Double model number, so Double algorithm can be used for calculation; While using a sorting algorithm, get a string of number, and calculate process involving an array. This algorithm is called Array type model; Matrix transpose meta-algorithm results lead to a Matrix. After operation, it is still the Matrix form, so it is a Matrix type model [3]. The metaalgorithm models are stored in the underlying model database and then with the help of a newly established new algorithm model. Make it in user model library. In the combination of GIS functions, data in data model is divided into preprocessing mathematical model, spatial analysis model and cartographic generalization model. The establishments of mathematical model libraries also need to consider the actual demand to support GIS algorithm.

\section{B. Logical organization structure design between models}

Through the logical classification of mathematical models within system, adopt object-oriented method to organize mathematical model in GIS management system. Mathematical model organization system structure is shown in Figure 1. Here, mathematical model is taken as an 
example. In the Figure, $\bigcirc$ represents the interface, the rest graphics represent entity class, abstract class graphics is expressed in italics,

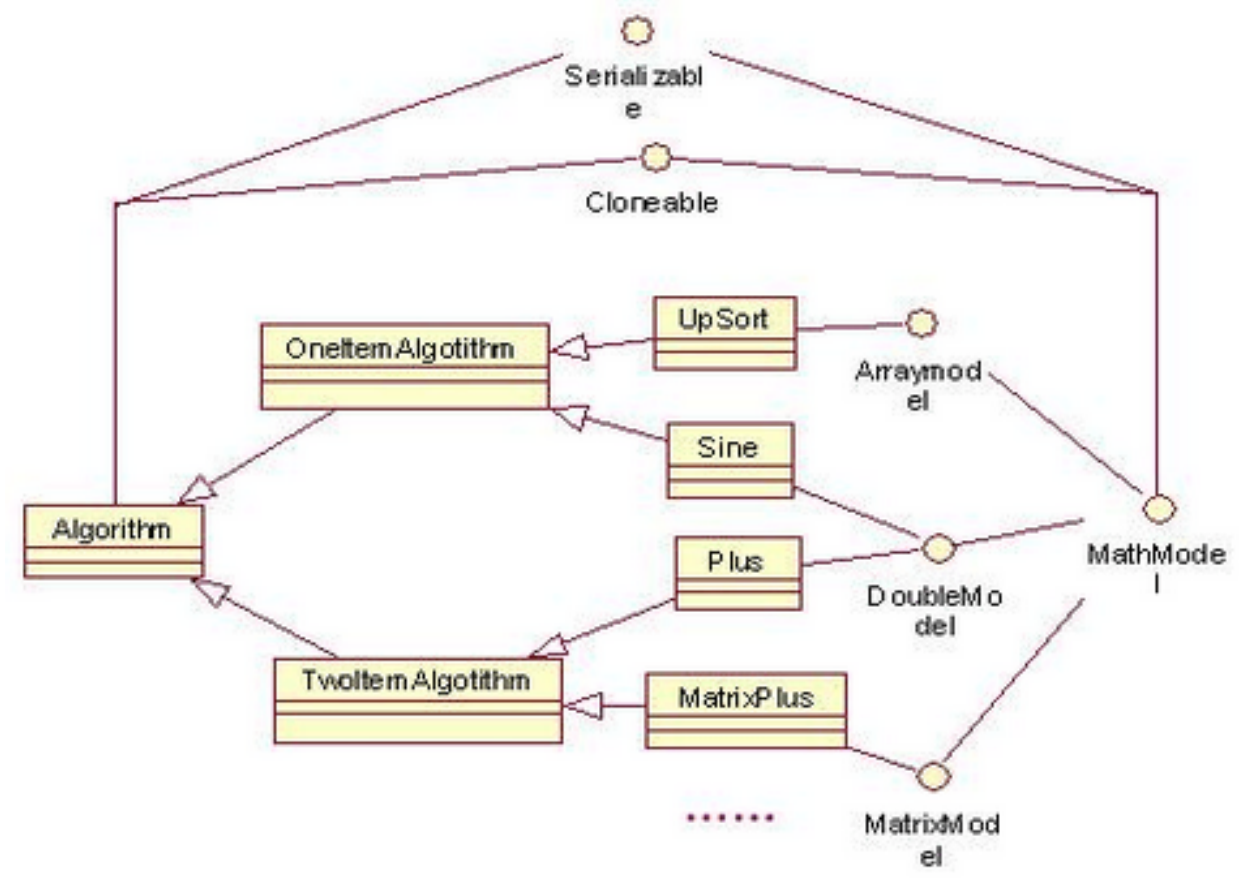

图 1 系统内模型之间的层次组织结构

From Figure 1, all of the mathematical models have connections with Serializable and Cloneable interfaces. They exist in set Java package. This package is very good to help the serialization and cloning of the mathematical model. Other model types can also call this package; established good models also can be transferred inwards or outwards in form of file. This has brought great convenience to the users [4]. By hierarchical graph, we can also see: there are two sets of structures in every meta-algorithm model: one is "interface" structure, and the other is "inherited base class" structure. These are all important elements of mathematical model. In the following, we will specifically describe interface design, base class design and the practice of constructing metaalgorithm model. 1. Interface design. From the figure, we can see that all models have a common interface, called MathModel. Then set up the corresponding interfaces according to the specific requirements. The detailed interface structure diagram is shown in Figure 2.

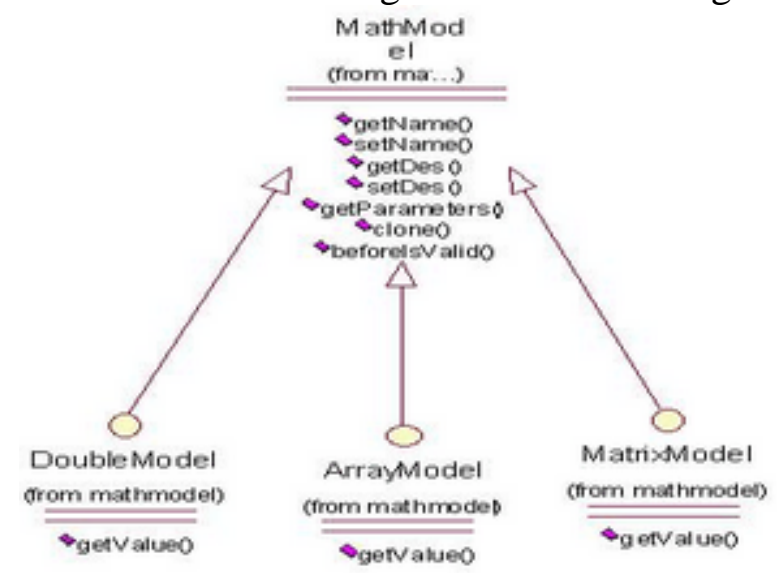

图 2 各层次接口的设计 
When design the base class, all the models also need to undertake indirectly corresponding abstract base class Algorithm, then combined with the actual operation required mesh, select the corresponding parent class inheritance.

\section{DYNAMIC GENERATION AND SYSTEM APPLICATION OF GIS MATHEMATICAL MODEL UNDER META-ALGORITHM}

\section{A. Dynamical generation of mathematical model}

Dynamically generate GIS mathematical model. We must make use of corresponding system interface. The mathematical models, under the condition of visualization, make their nesting, combination, and construct corresponding mathematical models. We will give specific and concrete build processes. Here take limit arithmetic classification model as an example. The mathematical formula is $A_{i}=L+(H-L) / M \times i(i=0, \ldots, M)$, in which $A i$ is the required $i$ class boundary, $H$ is the maximum of processing data, $\mathrm{L}$ is the minimum value in processing data, $\mathrm{M}$ is total class number. Preliminary subdivision of model should be in accordance with double linear thinking, then the model can be divided into $\mathrm{L}^{+}(\mathrm{H}-\mathrm{L}) / \mathrm{M} \times \mathrm{i}$ model and $(\mathrm{M}+1)$ dimensional array mathematical model $A[0,1,2 \ldots . . . M]$. After decomposing, two models can be further subdivided. Dimensional array mathematical model A need models like matrix model and array model, and $\mathrm{L}+(\mathrm{H}-\mathrm{L}) / \mathrm{M} \times \mathrm{i}$ model need more models, both with array maximum, minimum value models, also matrix multiplication and addition models, and so on. Then carry on visualization building, and according to Array type model provided by model library system to decompose the two mathematical models for modeling processing [5].

\section{B. GIS mathematical model database system application}

After designed GIS mathematical model database system, devote it to actual production application, and exert its function. Here thematic cartography teaching model is taken for example. Thematic data processing project elements classification mathematical model is an object. Take the production data of summer crops in Henan province to test and make grading thematic map in system model. The detailed integration application is as follows: 1 . Choose the required property fields in thematic mapping, and we choose attribute "area sowed" here. 2. Use "boundaries arithmetic classification model" in thematic mapping mathematical model database, as we mentioned above. 3. Configure variable parameters for the mathematical models, and the sown area field value is given as "pending data sources: A", assign value to the total series $\mathrm{M}$, value is 5.0, thus accomplish the thematic map in Henan province related summer crops planting area classification set. 4. Configure corresponding symbols for each level. Here call the model after the formation of grading thematic map [6]. The entire application process covers selection, call, settings, etc, and also uses "space geometric classification model" grading "production", thus draws the hierarchical graph total series set $\mathrm{M}=6.0$, geometric $\mathrm{q}=2.0$, and gets mathematical formula: $\mathrm{A}_{i}=\mathrm{L}+\left(1-\mathrm{q}^{i}\right) /\left(1-\mathrm{q}^{M}\right) \times(\mathrm{H}-\mathrm{L})$, and $\mathrm{q}$ is ratio.

\section{CONCLUSION}

Meta-algorithm is added to GIS mathematical model database system design to meet the new requirements for GIS development. Mathematical model library system under meta-algorithm has basically the same function and principle with that of former model mathematical library systems, but in the underlying model base, the organization and representation are different. To make the model improvement, the model has the advantages like fast, flexible and general. The algorithm optimization model system design should be actively and widely used in the actual research and development process of production. 


\section{REFERENCES}

[1] Jiang Nan, Zhang Lihong, Zhang Yajun, etc. GIS mathematical model library system design based on meta-algorithm [J]. Journal of Surveying and Mapping Science, 2008 (1) : 74-76.

[2] Zhang Lihong, Zhang Yajun, Dong Jingjing. Meta-algorithm GIS mathematical model database system design and implementation [C] 2009 China's geographic information industry BBS. 2009:18.

[3] Zhang Lihong, Jiang Nan, Zhang Yajun, etc. The establishment and application of mathematical special data processing calculations model library [J]. Journal of Surveying and Mapping Science and Technology, 2008, 25 (1) : 72-74,78.

[4] Yuan Chengjun. Overall planning decision support system research for land use at county level based on GIS ---- Take Guizhou, Qianxi as an example [D]. Guizhou University, 2008.

[5] Zhang Xiaonan. Qianjiang city land ecological environment quality evaluation and farmland classification research based on GIS [D]. Huazhong Agricultural University, 2006.

[6] Dai Changbiao. The overall design and key technology research of coal mine safety virtual reality simulation system [D]. China University of Geosciences (Wuhan), 2007. 